POS $\quad \begin{aligned} & \text { PROCEEDINGS } \\ & \text { OF SCIENCE }\end{aligned}$

\title{
First measurement of differential cross sections for ttbar production in dilepton final state at $13 \mathrm{TeV}$ collisions
}

\author{
Youn ROH*Korea University (KR) \\ E-mail: youn.jung.rohecern.ch \\ on behalf of the CMS Collaboration
}

Differential cross sections of top-quark pair production are measured in the dilepton decay channel with proton-proton collisions at a center-of-mass energy of $13 \mathrm{TeV}$. The measurement is performed with a Run II data sample using CMS detector at the Large Hadron Collider. In this analysis, we measure the differential cross sections with respect to kinematic variables of leptons, $\mathrm{b}$ jets, and top-quarks.

8th International Workshop on Top Quark Physics, TOP2015

14-18 September, 2015

Ischia, Italy

\footnotetext{
* Speaker.
} 


\section{Introduction}

Normalized differential cross sections for top quark pair productions are measured in the dilepton (electron or muon) decay channel ( shown in Figure 1 ) with proton-proton collisions at a center-of-mass energy of $13 \mathrm{TeV}$ using CMS experiment [1] in 2015.

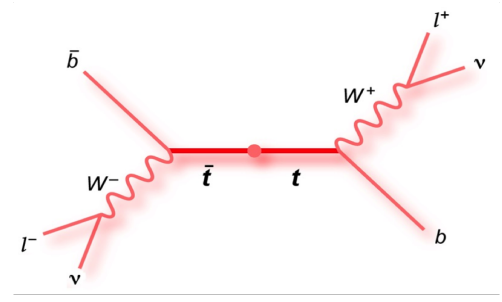

Figure 1: Feynman diagram of top-quark pair production decaying to lepton pairs. The $l^{ \pm}$symbol indecates a charged lepton.

\section{Data samples}

The measurement is done with data sample recorded in 2015 by CMS detector corresponding to an integrated luminosity of $42 \mathrm{pb}^{-1}$. We have used double lepton (electron or muon) triggered data samples and simulated samples which are generated by POWHEG, MG5_aMC@NLO and Madgraph, showered with either Pythia8 or Herwig++. The simulation includes $t \bar{t}+$ jets, Drell-Yan (DY), W+jets, single top quark production and dibosons (WW,ZZ,WZ)

\section{Event selection}

Figure 2 shows the distribution for the number of jets, jets identified as b's, and the transverse momentum of leptons and $b$ jets after applying the following event selection and before the kinematic reconstruction of the $t \bar{t}$ system.

- Electron/Muon : $P_{T}>20 \mathrm{GeV},|\eta|<2.4$

- Jet $: P_{T}>30 \mathrm{GeV},|\eta|<2.4$

- Opposite signed two leading $P_{T}$ leptons $(e e / \mu \mu / e \mu) M_{l l}>20 \mathrm{GeV}$, $\mathrm{Z}$ mass veto $\left(\left|M_{l l}-91\right|<15 \mathrm{GeV}\right)$ for $e e / \mu \mu$ where $M_{l l}$ is invariant mass of lepton pair.

- Missing transverse energy $Z_{T}>40 \mathrm{GeV}$ for $e e / \mu \mu, 2$ jets, $1 \mathrm{~b}$-tagged jet 

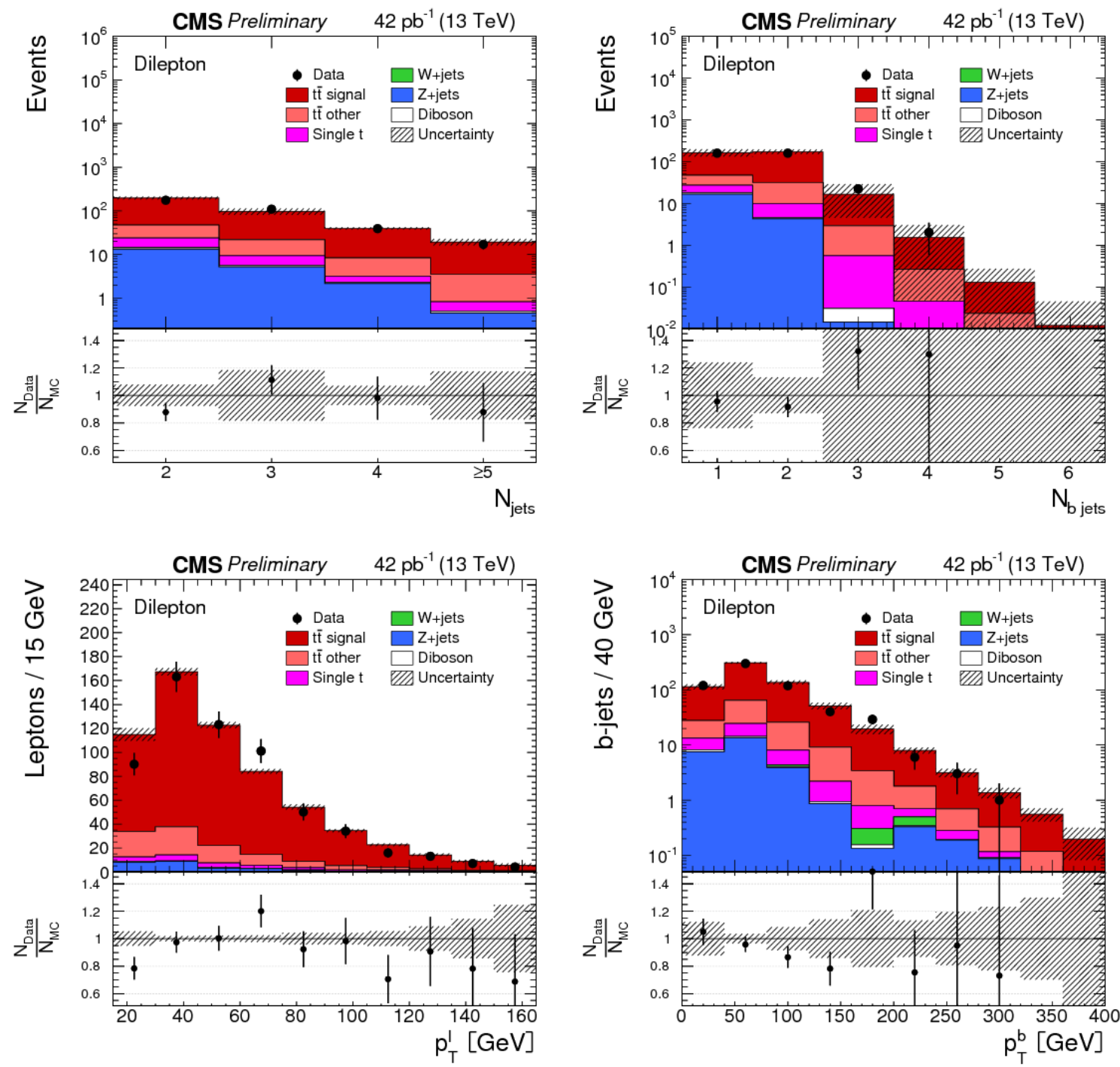

Figure 2: Kinematic distributions after event selection and before the kinematic reconstruction of the $t \bar{t}$ system. The hatched regions correspond to the shape uncertainties for the signal and backgrounds. The lower part of each plot shows the ratio of data to the predictions. [2]

\section{Top quark reconstruction}

The top quarks are determined from the four momenta of all final-state objects by means of an algebraic kinematic reconstruction method. The following constraints are imposed: the balance of $P_{T}$ of the two neutrinos; the $\mathrm{W}$ boson and the top quark mass. Effect of detector resolution is accounted for by studying the detector response for each object. The neutrino momenta are yielded with smallest invariant mass of the $t \bar{t}$ system. In case of ambiguity, the solution for the momenta of the neutrinos is chosen as the one which yields the lowest mass of the $t \bar{t}$ system. For each solution, a weight is calculated based on the expected true invariant mass spectrum of the lepton and $b$ jet. 
Over 100 reconstruction attempts, the top quarks are calculated as a weighted average.

\section{Normalized differential cross section}

Normalized differential $t \bar{t}$ cross section is measured as a function of jet multiplicity and of kinematic variables of top quark and $t \bar{t}$ system, as follows:

$$
\frac{1}{\sigma} \frac{d \sigma_{i}}{d X}=\frac{1}{\sigma} \frac{x_{i}}{\Delta_{i} X}
$$

where $x_{i}$ is the number of signal events measured in data after background subtraction and corrected for detector efficiencies, acceptances, and migrations. The correction is done using a regularized unfolding method $\left(\Sigma_{j} A_{i j}-1\left[N_{D a t a, j}-N_{b k, j}\right]\right.$ where A : response matrix of kinematic variables, $N_{\text {data } / b k}$ : number of data/estimated background). $\Delta_{i} X$ is the bin width. $\sigma$ is total cross section. $X$ represents a variable such as the jet multiplicity, top $P_{T}$, top rapidity, $t \bar{t} P_{T}$ [2].

\section{Result}

The normalized differential cross section as a function of the jet multiplicity is determined at the particle level, where the physics objects are defined as follows: $P_{T}^{l}>20 \mathrm{GeV}, P_{T}^{j}>20 \mathrm{GeV}$, $|\eta|<2.4$. The top quark and $t \bar{t}$ system observables are defined with respect to the top quarks or antiquarks before the decay (parton level) and after QCD radiation, and extrapolated to the full phase space (See Figure 3).

\section{Conclusion}

Normalized differential cross sections for top quark pair production are measured in dilepton decay channel with respect to the jet multiplicity, top $P_{T}$, top rapidity, $t \bar{t} P_{T}$. The measurements are compared to predictions from POWHEG + PYTHIA8, MG5 aMC@ NLO + PYTHIA8, MADGRAPH + PYTHIA8, and POWHEG + HERWIG++. The measurements are in agreement with standard model predictions.

\section{References}

[1] CMS Collaboration, "The CMS experiment at the CERN LHC”, JINST 3 (2008) S08004

[2] CMS Collaboration, "First measurement of the differential cross section for $t \bar{t}$ production in the dilepton final state at $\sqrt{s}=13$ TeV", CMS-PAS-TOP-15-010 (2015) 

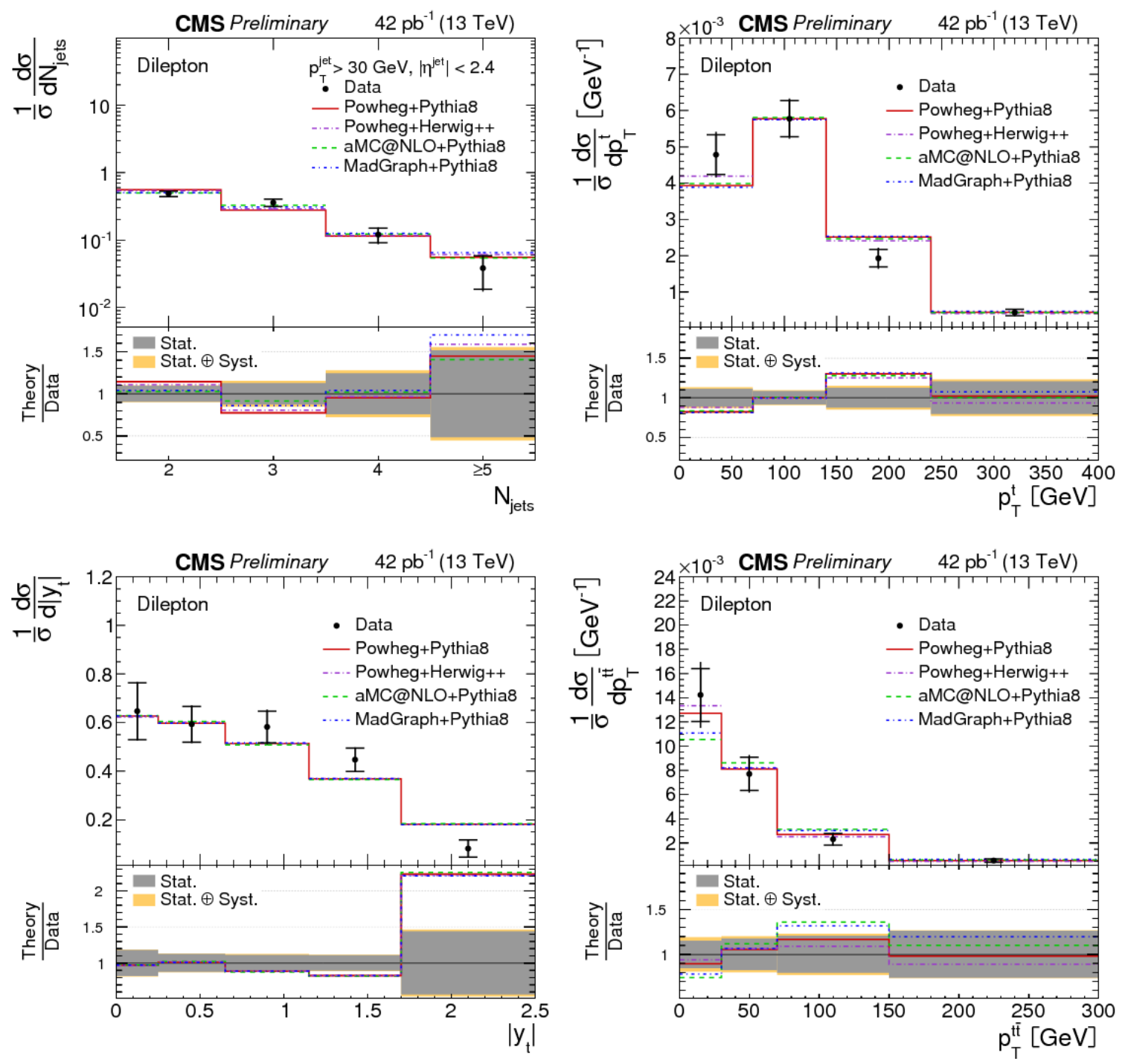

Figure 3: Normalized differential $t \bar{t}$ production cross section as a function of the jet multiplicity, top $P_{T}$, top rapidity, $t \bar{t} P_{T}$. The inner (outer)error bars indicate the statistical (combined statistical and systematic) uncertainty. The measurements are compared to predictions from POWHEG + PYTHIA8, MG5 aMC@NLO + PYTHIA8, MADGRAPH + PYTHIA8, and POWHEG + HERWIG++. [2] 